\title{
Computational Intelligence in Data Mining
}

\author{
Janos Abonyi and Ferenc Szeifert \\ University of Veszprem, Department of Process Engineering, \\ P.O. Box 158, H-8201 Veszprem, Hungary, abonyij@fmt.vein.hu \\ www.fmt.vein.hu/softcomp
}

\begin{abstract}
This paper describes links between computational intelligence (CI), data mining and knowledge discovery. The generating elements of soft computing based data mining algorithms are defined where the extracted knowledge is represented by fuzzy rule-based expert systems. It is recognized that both model performance and interpretability are of major importance, and effort has to make to keep the resulting rule bases small and comprehensible. Therefore, the model is derived from data by CI techniques developed for feature selection, feature extraction, model optimization and model reduction (rule base simplification). Applications of these generating elements to the Wine data classification problem are also shown.
\end{abstract}

\section{Introduction}

In our society the amount of data doubles almost every year. Hence, there is an urgent need for a new generation of computational techniques and tools to assist humans in extracting useful information (knowledge) from the rapidly growing volumes of data.

When we attempt to solve realworld problems, like extracting knowledge from large amount of data, we realize that they are typically illdefined systems, difficult to model and with largescale solution spaces. In these cases, precise models are impractical, too expensive, or nonexistent. Furthermore, the relevant available information is usually in the form of empirical prior knowledge and inputoutput data representing instances of the system's behavior. Therefore, we need an approximate reasoning systems capable of handling such imperfect information. Computational Intelligence (CI) and Soft Computing (SC) are recently coined terms describing the use of many emerging computing disciplines. According to Zadeh (1994): "... in contrast to traditional, hard computing, soft computing is tolerant of imprecision, uncertainty, and partial truth.” In this context Fuzzy Logic (FL), Probabilistic Reasoning (PR), Neural Networks (NNs), and Genetic Algorithms (GAs) are considered as main components of CI. Each of these technologies provide us with complementary reasoning and searching methods to solve complex, realworld problems. What is important to note is that soft computing is not a melange. Rather, it is a partnership in which each of the partners contributes a distinct methodology for addressing problems in its domain. In this perspective, the principal constituent methodologies in CI are complementary rather than competitive.

The aim of this paper is to show how these elements of CI can be used in data mining. 
The remainder of this article is organized as follows. Section 2 explains the steps of knowledge discovery including methods and algorithms of data mining. In this section an overview is given how CI techniques can be applied in these steps. In the remaining sections, tools for visualization, knowledge representation, classifier identification and reduction are discussed. The proposed approaches are experimentally evaluated for the three-class Wine classification problem. Finally, conclusions are given in Section 8.

\section{Knowledge Discovery and Data Mining}

Historically the notion of finding useful patterns in data has been given a variety of names including data mining, knowledge extraction, information discovery, and data pattern processing. The term data mining has been mostly used by statisticians, data analysts, and the management information systems (MIS) communities.

The term knowledge discovery in databases (KDD) refers to the overall process of discovering knowledge from data, while data mining refers to a particular step of this process. Data mining is the application of specific algorithms for extracting patterns from data [1]. The additional steps in the KDD process, such as data selection, data cleaning, incorporating appropriate prior knowledge, and proper interpretation of the results are essential to ensure that useful knowledge is derived form the data.

KDD has evolved from the intersection of research fields such as machine learning, pattern recognition, databases, statistics, artificial intelligence, and more recently it gets new inspiration from computational intelligence.

\subsection{Steps of Knowledge Discovery}

Brachman and Anand [2] give a practical view of the KDD process emphasizing the interactive nature of the process. Here we broadly outline some of its basic steps depicted in Figure 1 taken from [3], and we show the connections of these steps to CI based models and algorithms.

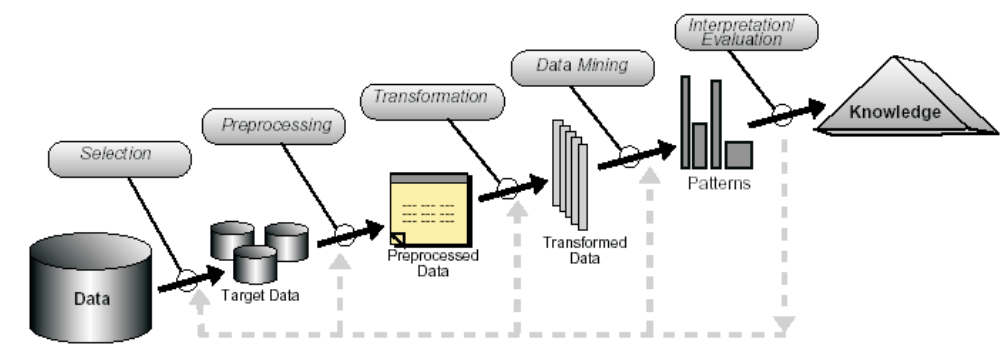

Fig. 1. Steps of the knowledge discovery process. 
1. Developing and understanding of the application domain and the relevant prior knowledge, and identifying the goal of the KDD process. The transparency of fuzzy systems allows the user to effectively combine different types of information, namely linguistic knowledge, first-principle knowledge and information from data. An example for the incorporation of prior knowledge into data-driven identification of dynamic fuzzy models of the Takagi-Sugeno type can be found in [4] where the prior information enters to the model through constraints defined on the model parameters. In [5] and [6] a different approach has been developed which uses block-oriented fuzzy models.

2. Creating target data set.

3. Data cleaning an preprocessing: basic operations such as the removal of noise, handling missing data fields.

4. Data reduction and projection: finding useful features to represent the data depending the goal of the task. Using dimensionality reduction or transformation methods to reduce the effective number of variables under consideration or to find invariant representation of data. Neural networks [7], cluster analysis [8], and neuro-fuzzy systems are often used for this purpose.

5. Matching the goals of the KDD process to a particular data mining method: Although the boundaries between prediction and description are not sharp, the distinction is useful for understanding the overall discovery goal. The goals of data mining are achieved via the following data mining methods:

- Clustering: Identification a finite set of categories or clusters to describe the data. Closely related to clustering is the method of probability density estimation. Clustering quantizes the available input-output data to get a set of prototypes and use the obtained prototypes (signatures, templates, etc., and many writers refer to as codebook) and use the prototypes as model parameters.

- Summation: finding a compact description for subset of data, e.g. the derivation of summary for association of rules and the use of multivariate visualization techniques.

- Dependency modeling: finding a model which describes significant dependencies between variables (e.g. learning of belief networks).

- Regression: learning a function which maps a data item to a real-valued prediction variable and the discovery of functional relationships between variables.

- Classification: learning a function that maps (classifies) a data item into one of several predefined classes.

- Change and Deviation Detection: Discovering the most significant changes in the data from previously measured or normative values. 
6. Choosing the data mining algorithm(s): selecting algorithms for searching for patterns in the data. This includes deciding which model and parameters may be appropriate and matching a particular algorithm with the overall criteria of the KDD process (e.g. the end-user may be more interested in understanding the model than its predictive capabilities.) One can identify three primary components in any data mining algorithm: model representation, model evaluation, and search.

- Model representation: the language is used to describe the discoverable patterns. If the representation is too limited, then no a amount of training time or examples will produce an accurate model for the data. Note that more powerful representation power of models increases the danger of overfitting the training data resulting in reduced prediction accuracy on unseen data. It is important that a data analysts fully comprehend the representational assumptions which may be inherent in a particular method.

For instance, rule-based expert systems are often applied to classification problems in fault detection, biology, medicine etc. Among the wide range of CI techniques, fuzzy logic improves classification and decision support systems by allowing the use of overlapping class definitions and improves the interpretability of the results by providing more insight into the classifier structure and decision making process [9]. In Section 3. a detailed discussion about the use of fuzzy techniques for knowledge representation in classifier systems will be given.

- Model evaluation criteria: qualitative statements or fit functions of how well a particular pattern (a model and its parameters) meet the goals of the KDD process. For example, predictive models can often judged by the empirical prediction accuracy on some test set. Descriptive models can be evaluated evaluated along the dimensions of predictive accuracy, novelty, utility, and understandability of the fitted model.

Traditionally, algorithms to obtain classifiers have focused either on accuracy or interpretability. Recently some approaches to combining these properties have been reported; fuzzy clustering is proposed to derive transparent models in [10], linguistic constraints are applied to fuzzy modeling in [9] and rule extraction from neural networks is described in [11]. Hence, to obtain compact and interpretable fuzzy models model reduction algorithms have to be used that will be overviewed in Section 4.

- Search method: consists of two components: parameter search and model search. Once the model representation and the model evaluation criteria are fixed, then the data mining problem has been reduced to purely an optimization task: find the parameters/models for the selected family which optimize the evaluation criteria given observed data and fixed model representation. Model search occurs as a loop over the parameters search method.

The automatic determination of fuzzy classification rules from data has been approached by several different techniques: neuro-fuzzy methods [12], genetic-algorithm based rule selection [13] and fuzzy clustering in combination with GA-optimization [14]. For high-dimensional classification 
problems, the initialization step of the identification procedure of the fuzzy model becomes very significant. Several CI based tools developed for this purpose will be presented in Section 5 .

7. Data mining: searching for patterns of interest in a particular representation form or a set of such representations: classification rules or trees, regression. Some of the CI models lend themselves to transform into other model structure that allows information transfer between different models. For example, in [15] a decision tree was mapped into a feedforward neural network. A variation of this method is given in [16] where the decision tree was used for the input domains discretization only. This approach was extended with a model pruning method in [17]. Another example is that as radial basis functions (RBF) are functionally equivalent to fuzzy inference systems $[18,19]$, tools for developed for the identification of RBFs can also be used to design fuzzy models.

8. Interpreting mined patterns, possibly return to any of steps 1-7 for further iteration. This step can also involve the visualization of the extracted patterns/models, or visualization of the data given the extracted models. Self-Organizing Map (SOM) as a special clustering tool provides a compact representation of the data distribution, hence it has been widely applied in the visualization of highdimensional data [20]. In section Section 6 the theory and in Section 7 the application of SOM will be presented.

9. Consolidating discovered knowledge: incorporating this knowledge into another system for further action, or simply documenting and reporting it.

\section{Effective Model Representation by Fuzzy Systems}

\subsection{Classifier Systems}

The identification of a classifier system means the construction of a model that predicts whether a given pattern, $\mathbf{x}_{k}=\left[x_{1, k}, \ldots, x_{n, k},\right]$, in which $y_{k}=\left\{c_{1}, \ldots, c_{C}\right\}$ class should be classified. The classic approach for this problem with $C$ classes is based on Bayes' rule. The probability of making an error when classifying an example $\mathbf{x}$ is minimized by Bayes' decision rule of assigning it to the class with the largest posterior probability:

$$
\mathbf{x} \text { is assigned to } c_{i} \Longleftrightarrow p\left(c_{i} \mid \mathbf{x}\right) \geq p\left(c_{j} \mid \mathbf{x}\right) \forall j \neq i
$$

The a posteriori probability of each class given a pattern $\mathbf{x}$ can be calculated based on the $p\left(\mathbf{x} \mid c_{i}\right)$ class conditional distribution, which models the density of the data belonging to the $c_{i}$ class, and the $P\left(c_{i}\right)$ class prior, which represents the probability that an arbitrary example out of data belongs to class $c_{i}$

$$
p\left(c_{i} \mid \mathbf{x}\right)=\frac{p\left(\mathbf{x} \mid c_{i}\right) P\left(c_{i}\right)}{p(\mathbf{x})}=\frac{p\left(\mathbf{x} \mid c_{i}\right) P\left(c_{i}\right)}{\sum_{j=1}^{C} p\left(\mathbf{x} \mid c_{j}\right) P\left(c_{j}\right)}
$$

As (1) can be rewritten using the numerator of (2) we would have an optimal classifier if we would perfectly estimate the class priors and the class conditional densities. Of course in practice one need to find approximate estimates of these quantities 
on a finite set of training data $\left\{\mathbf{x}_{k}, y_{k}\right\}, k=1, \ldots, N$. Priors $P\left(c_{i}\right)$ are often estimated on the basis of the training set as the proportion of samples of class $c_{i}$ or using prior knowledge. The $p\left(c_{i} \mid \mathbf{x}\right)$ class conditional densities can be modeled with nonparametric methods like histograms, nearest-neighbors or parametric methods such as mixture models.

\subsection{Fuzzy Rules for Providing Interpretability of Classifiers}

The classical fuzzy rule-based classifier consists of fuzzy rules that each describe one of the $C$ classes. The rule antecedent defines the operating region of the rule in the $n$-dimensional feature space and the rule consequent is a crisp (non-fuzzy) class label from the $\left\{c_{1}, \ldots, c_{C}\right\}$ set:

$$
r_{i}: \text { If } x_{1} \text { is } A_{i, 1}\left(x_{1, k}\right) \text { and } \ldots x_{n} \text { is } A_{i, n}\left(x_{n, k}\right) \text { then } \hat{y}=c_{i}, \quad\left[w_{i}\right]
$$

where $A_{i, 1}, \ldots, A_{i, n}$ are the antecedent fuzzy sets and $w_{i}$ is a certainly factor that represents the desired impact of the rule. The value of $w_{i}$ is usually chosen by the designer of the fuzzy system according to his or her belief in the accuracy of the rule. When such knowledge is not available, $w_{i}=1, \forall i$ is used.

The and connective is modeled by the product operator allowing for interaction between the propositions in the antecedent. Hence, the degree of activation of the $i$ th rule is calculated as:

$$
\beta_{i}\left(\mathbf{x}_{k}\right)=w_{i} \prod_{j=1}^{n} A_{i, j}\left(x_{j, k}\right)
$$

The output of the classical fuzzy classifier is determined by the winner takes all strategy, i.e. the output is the class related to the consequent of the rule that has the highest degree of activation:

$$
\hat{y}_{k}=c_{i}^{*}, i^{*}=\underset{1 \leq i \leq C}{\arg \max } \beta_{i}\left(\mathbf{x}_{k}\right)
$$

The fuzzy classifier defined by the previous equations is in fact a quadratic Bayes classifier when $\beta_{i}\left(\mathbf{x}_{k}\right)=p\left(\mathbf{x} \mid c_{i}\right) P\left(c_{i}\right)$.

As the number of the rules in the above representation is equal to the number of the classes, the application of this classical fuzzy classifier is restricted. In the [21], a new rule-structure has been derived to avoid this problem, where the $p\left(c_{i} \mid \mathbf{x}\right)$ posteriori densities are modeled by $R>C$ mixture of models

$$
p\left(c_{i} \mid \mathbf{x}\right)=\sum_{l=1}^{R} p\left(r_{l} \mid \mathbf{x}\right) P\left(c_{i} \mid r_{l}\right)
$$

This idea results in fuzzy rulebase where the consequent of rule defines the probability of the given rule represents the $c_{1}, \ldots, c_{C}$ classes:

$$
\begin{gathered}
r_{i}: \text { If } x_{1} \text { is } A_{i, 1}\left(x_{1, k}\right) \text { and } \ldots x_{n} \text { is } A_{i, n}\left(x_{n, k}\right) \text { then } \\
\hat{y}_{k}=c_{1} \text { with } P\left(c_{1} \mid r_{i}\right) \ldots, \hat{y}_{k}=c_{C} \text { with } P\left(c_{C} \mid r_{i}\right)\left[w_{i}\right]
\end{gathered}
$$

The aim of the remaining part of the paper is to review some technique for the identification of the fuzzy classifier presented above. In addition, methods for reduction of the model will be described. 


\section{Model Evaluation Criteria and Rule Base Reduction}

Traditionally, algorithms to obtain classifiers have been focused either on accuracy or interpretability. Recently some approaches to combining these properties have been reported; fuzzy clustering is proposed to derive transparent models in [10], linguistic constraints are applied to fuzzy modeling in [9] and rule extraction from neural networks is described in [11].

\subsection{Similarity-driven rule base simplification}

The similarity-driven rule base simplification method [22] uses a similarity measure to quantify the redundancy among the fuzzy sets in the rule base. A similarity measure based on the set-theoretic operations of intersection and union is applied:

$$
S\left(A_{i, j}, A_{l, j}\right)=\frac{\left|A_{i, j} \cap A_{l, j}\right|}{\left|A_{i, j} \cup A_{l, j}\right|}
$$

where $|$.$| denotes the cardinality of a set, and the \cap$ and $\cup$ operators represent the intersection and union of fuzzy sets, respectively. $S$ is a symmetric measure in $[0,1]$. If $S\left(A_{i, j}, A_{l, j}\right)=1$, then the two membership functions $A_{i, j}$ and $A_{l, j}$ are equal. $S\left(A_{i, j}, A_{l, j}\right)$ becomes 0 when the membership functions are non-overlapping. The complete rule base simplification algorithm is given in [22].

Similar fuzzy sets are merged when their similarity exceeds a user defined threshold $\theta \in[0,1](\theta=0.5$ is applied). Merging reduces the number of different fuzzy sets (linguistic terms) used in the model and thereby increases the transparency. The similarity measure is also used to detect "don't care" terms, i.e., fuzzy sets in which all elements of a domain have a membership close to one. If all the fuzzy sets for a feature are similar to the universal set, or if merging led to only one membership function for a feature, then this feature is eliminated from the model. The method is illustrated in Figure 2

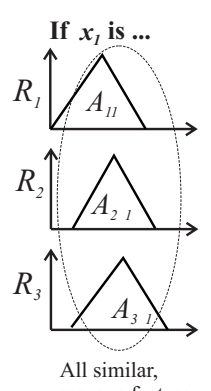
remove feature.
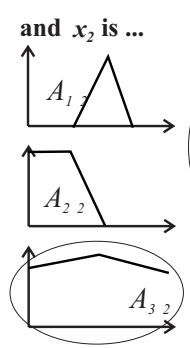

Similar to universe, Merge similar sets. remove set.
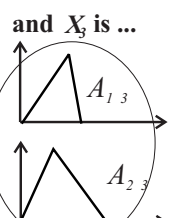
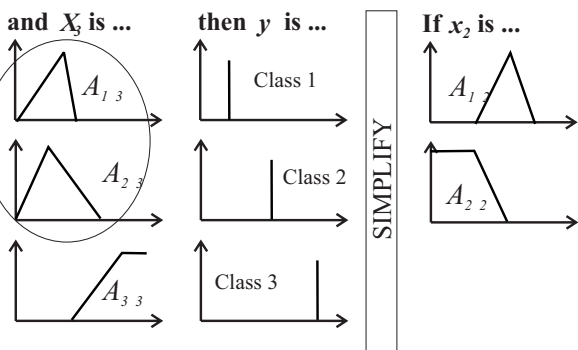

Fig. 2. Similarity-driven simplification. 


\subsection{Multi-Objective Function for GA based Identification}

To improve the classification capability of the rule base, genetic algorithm (GA) optimization method can be applied [23] where the cost function is based on the model accuracy measured in terms of the number of misclassifications. Also other model properties can be optimized by applying multi-objective functions. For example in [24] to reduce the model complexity, the misclassification rate is combined with a similarity measure in the GA objective function. Similarity is rewarded during the iterative process, that is, the GA tries to emphasize the redundancy in the model. This redundancy is then used to remove unnecessary fuzzy sets in the next iteration. In the final step, fine tuning is combined with a penalized similarity among fuzzy sets to obtain a distinguishable term set for linguistic interpretation.

The GAs is subject to minimize the following multi-objective function:

$$
J=\left(1+\lambda S^{*}\right) \cdot \text { Error }
$$

where $S^{*} \in[0,1]$ is the average of the maximum pairwise similarity that is present in each input, i.e., $S^{*}$ is an aggregated similarity measure for the total model. The weighting function $\lambda \in[-1,1]$ determines whether similarity is rewarded $(\lambda<0)$ or penalized $(\lambda>0)$.

\subsection{Other Reduction Algorithms}

The application of orthogonal transforms for reducing the number of rules has received much attention in recent literature [25]. These methods evaluate the output contribution of the rules to obtain an importance ordering. For modeling purpose Orthogonal Least Squares (OLS) is the most appropriate tool [26]. Evaluating only the approximation capabilities of the rules, the OLS method often assigns high importance to a set of redundant or correlated rules. To avoid this, in [27] some extension for the OLS method was proposed.

Using too many input variables may result in difficulties in the interpretability capabilities of the obtained classifier. Hence, selection of the relevant features is usually necessary. Others have focused on reducing the antecedent by similarity analysis of the fuzzy sets [24], however this method is not very suitable for feature selection. Hence, for this purpose, Fischer interclass separability method which is based on statistical properties of the data [28] has been modified in [29].

\section{CI based Search Methods for the Identification of Fuzzy Classifiers}

Fixed membership functions are often used to partition the feature space [13]. Membership functions derived from the data, however, explain the data-patterns in a better way. The automatic determination of fuzzy classification rules from data has been approached by several different techniques: neuro-fuzzy methods [12], genetic-algorithm based rule selection [13] and fuzzy clustering in combination with 
GA-optimization [14]. For high-dimensional classification problems, the initialization step of the identification procedure of the fuzzy model becomes very significant. Common initializations methods such as grid-type partitioning [13] and rule generation on extrema initialization [30], result in complex and non-interpretable initial models and the rule-base simplification and reduction step become computationally demanding.

\subsection{Identification by Fuzzy Clustering}

To obtain compact initial fuzzy models fuzzy clustering algorithms [14] or similar but less complex covariance based initialization techniques [31] were put forward, where the data is partitioned by ellipsoidal regions (multivariable mebership functions). Normal fuzzy sets can then be obtained by an orthogonal projection of the multivariable membership functions onto the input-output domains. The projection of the ellipsoids results in hyperboxes in the product space. The information loss at this step makes the model suboptimal resulting in a much worse performance than the initial model defined by multivariable membership functions. However, gaining linguistic interpretability is the main advantage derived from this step. To avoid the erroneous projection step mulativariate membership functions [32] or clustering algorithms providing axis-parallel clusters can be used [21]

\subsection{Other Initialization Algorithms}

For the effective initialization of fuzzy classifiers crisp decision tree-based initialization technique is proposed in [33]. DT-based classifiers perform a rectangular partitioning of the input space, while fuzzy models generate non-axis parallel decision boundaries [34]. Hence, the main advantage of rule-based fuzzy classifiers over crisp-DTs is the greater flexibility of the decision boundaries. Therefore fuzzy classifiers can be more parsimonious than DTs and one may conclude that the fuzzy classifiers, based on the transformation of DTs only [35], [36] will usually be more complex than necessary. This suggest that the simple transformation of a DT into a fuzzy model may be successfully followed by model reduction steps to reduce the complexity and improve the interpretability. The next section proposes rule-base optimization and simplification steps for this purpose.

\section{Clustering by SOM for Visualization}

The Self-Organizing Map (SOM) algorithm performs a topology preserving mapping from high dimensional space onto map units so that relative distances between data points are preserved. The map units, or neurons, form usually a two dimensional regular lattice. Each neuron $i$ of the SOM is represented by an $l$-dimensional weight, or model vector $\mathbf{m}_{i}=\left[m_{i, 1}, \ldots, m_{i, l}\right]^{T}$. These weigh vectors of the SOM form a codebook. The neurons of the map are connected to adjacent neurons by a neighborhood relation, which dictates the topology of the map. The number of the 
neurons determines the granularity of the mapping, which affects the accuracy and the generalization capability of the SOM.

SOM is a vector quantizer, where the weights play the role of the codebook vectors. This means, each weigh vector represents a local neighborhood of the space, also called Voronoi cell. The response of a SOM to an input $\mathbf{x}$ is determined by the reference vector (weight) $\mathbf{m}_{i}^{0}$ which produces the best match of the input

$$
i^{0}=\underset{i}{\arg \min }\left\|\mathbf{m}_{i}-\mathbf{x}\right\|
$$

where $i^{0}$ represents the index of the Best Matching Unit (BMU).

During the iterative training of SOM, the SOM forms an elastic net that folds onto "cloud" formed by the data. The net tends for approximate the probability density of the data: the codebook vectors tend to drift there where the data are dense, while there are only a few codebook vectors where the data are sparse. The training of SOM can be accomplished generally with a competitive learning rule as

$$
\mathbf{m}_{i}^{(k+1)}=\mathbf{m}_{i}^{(k)}+\eta \Lambda_{i^{0}, i}\left(\mathbf{x}-\mathbf{m}_{i}^{(k)}\right)
$$

where $\Lambda_{i^{0}, i}$ is a spatial neighborhood function and $\eta$ is the learning rate. Usually, the neighborhood function is

$$
\Lambda_{i^{0}, i}=\exp \left(\frac{\left\|\mathbf{r}_{i}-\mathbf{r}_{i}^{0}\right\|^{2}}{2 \sigma^{2(k)}}\right)
$$

where $\left\|\mathbf{r}_{i}-\mathbf{r}_{i}^{0}\right\|$ represents the Euclidean distance in the output space between the $i$-th vector and the winner.

\section{Case study: Wine Classification by CI techniques}

\subsection{Wine Data}

The Wine data ${ }^{1}$ contains the chemical analysis of 178 wines grown in the same region in Italy but derived from three different cultivars. The problem is to distinguish the three different types based on 13 continuous attributes derived from chemical analysis. : Alcohol, Malic acid, Ash, Alcalinity of ash, Magnesium, Total phenols, Flavanoids, Nonflavanoids phenols, Proanthocyaninsm color intensity, Hue, OD280/OD315 of dilluted wines and Proline (Figure 3).

\subsection{Fuzzy Classifier Identified by GA}

An initial classifier with three rules was constructed by the covariance-based model initialization technique proposed in [29] using all samples resulting in $90.5 \%$ correct, $1.7 \%$ undecided and $7.9 \%$ misclassifications for the three wine classes. Improved classifiers are developed based the GA based optimization technique discussed in Section 4.2. Based on the similarity analysis of the optimized fuzzy sets,

\footnotetext{
${ }^{1}$ The Wine data is available from the University of California, Irvine, via anonymous ftp ftp.ics.uci.edu/pub/machine-learning-databases.
} 


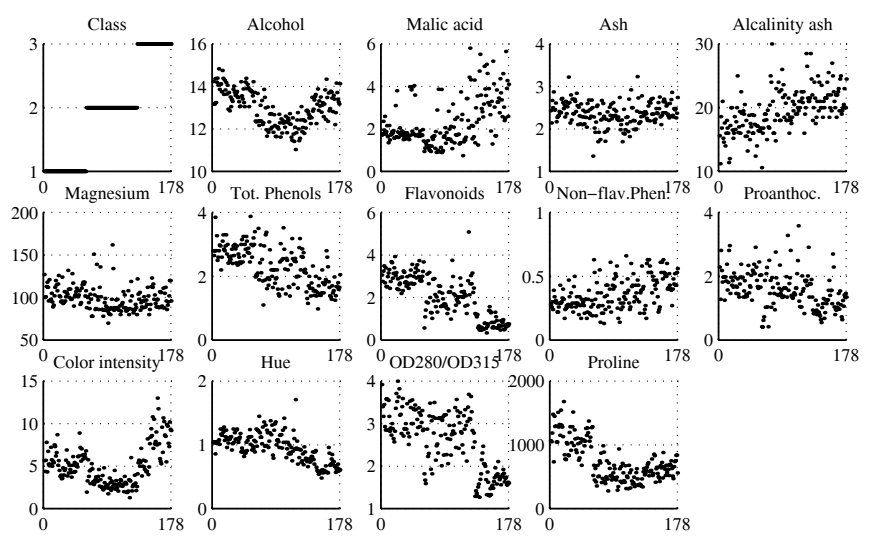

Fig. 3. Wine data: 3 classes and 13 attributes.

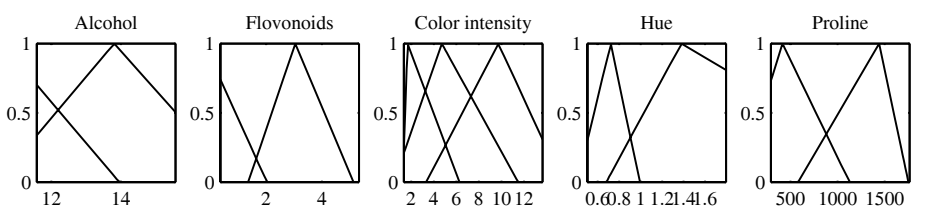

Fig. 4. The fuzzy sets of the optimized three rule classifier for the Wine data.

some features have been removed from individual rules, while the interclass separability method have been used to omit some features in all the rules. The achieved membership functions are shown in Figure 4, while the obtained rules are shown in Table 1.

\subsection{Fuzzy Classifier Identified by Fuzzy Clustering}

A fuzzy classifier, that utilizes all the 13 information profile data about the wine, has been identified by the clustering algorithm proposed in [21], where the obtained classiffier is formulated by rules given by (8). Fuzzy models with three and four rules were identified. The three rule-model gave only 2 misclassification $(98.9 \%)$. When a cluster was added to improve the performance of this model, the obtained classifier gave only 1 misclassification $(99.4 \%)$.

The classification power of the identified models is compared with fuzzy models with the same number of rules obtained by Gath-Geva clustering, as Gath-Geva clustering can be considered the unsupervised version of the proposed clustering algorithm. The Gath-Geva identified fuzzy model gives 8 (95.5\%) misclassification

Table 1. Three rule fuzzy classifier ( $\mathrm{L}=\mathrm{low}, \mathrm{M}=$ medium , $\mathrm{H}=$ high).

$\begin{array}{lllllllllllll}1 & 2 & 3 & 4 & 5 & 6 & 7 & 8 & 9 & 10 & 11 & 12 & 13\end{array}$

Alc Mal Ash aAsh Mag Tot Fla nFlav Pro Col Hue OD2 Pro Class

\begin{tabular}{lllllllllllllll}
\hline$R_{1}$ & $\mathrm{H}$ & - & - & - & - & - & $\mathrm{H}$ & - & - & $\mathrm{M}$ & $\mathrm{L}$ & - & $\mathrm{L}$ & 1 \\
$R_{2}$ & $\mathrm{~L}$ & - & - & - & - & - & - & - & - & $\mathrm{L}$ & $\mathrm{L}$ & - & $\mathrm{H}$ & 2 \\
$R_{3} \mathrm{H}$ & - & - & - & - & - & $\mathrm{L}$ & - & - & $\mathrm{H}$ & $\mathrm{H}$ & - & $\mathrm{H}$ & 3 \\
\hline
\end{tabular}


when the fuzzy model has three rules and $6(96.6 \%)$ misclassification with four rules. These results indicate that the proposed clustering method effectively utilizes the class labels.

The interclass separability based model reduction technique is applied to remove redundancy and simplify the obtained fuzzy models and five features were selected. The clustering has been applied again to identify a model based on the selected five attributes. This compact model with three, four and five rules gives four, two and zero misclassification, respectively. The resulted membership functions and the selected features are shown in Figure 5.

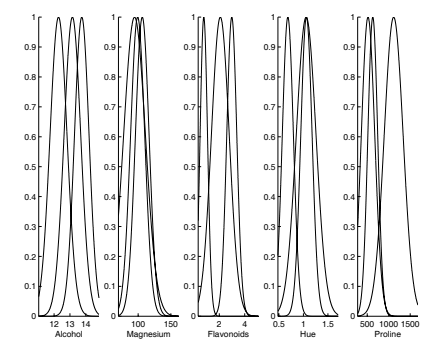

Fig. 5. Membership functions obtained by fuzzy clustering.

\subsection{Visualization by SOM}

The SOM presented in Section 6. has been utilized to visualize the Wine data. SOM can be effectively used for correlation hunting, which procedure is useful for detecting the redundant features. It is interesting to note that the rules given in Table 1 can easily validated by the map of the variables given in Figure 6

\subsection{Discussion}

The Wine data is widely applied for comparing the capabilities of different data mining tools. Corcoran and Sen [37] applied all the 178 samples for learning 60 non-fuzzy if-then rules in a real-coded genetic based-machine learning approach. They used a population of 1500 individuals and applied 300 generations, with full replacement, to come up with the following result for ten independent trials: best classification rate $100 \%$, average classification rate $99.5 \%$ and worst classification rate $98.3 \%$ which is 3 misclassifications. Ishibuchi et al. [13] applied all the 178 samples designing a fuzzy classifier with 60 fuzzy rules by means of an integercoded genetic algorithm and grid partitioning. Their population contained 100 individuals and they applied 1000 generations, with full replacement, to come up with the following result for ten independent trials: best classification rate $99.4 \%$ (1 misclassifications), average classification rate $98.5 \%$ and worst classification rate $97.8 \%$ (4 misclassifications). In both approaches the final rule base contains 60 rules. The main difference is the number of model evaluations that was necessary to come to the final result. 


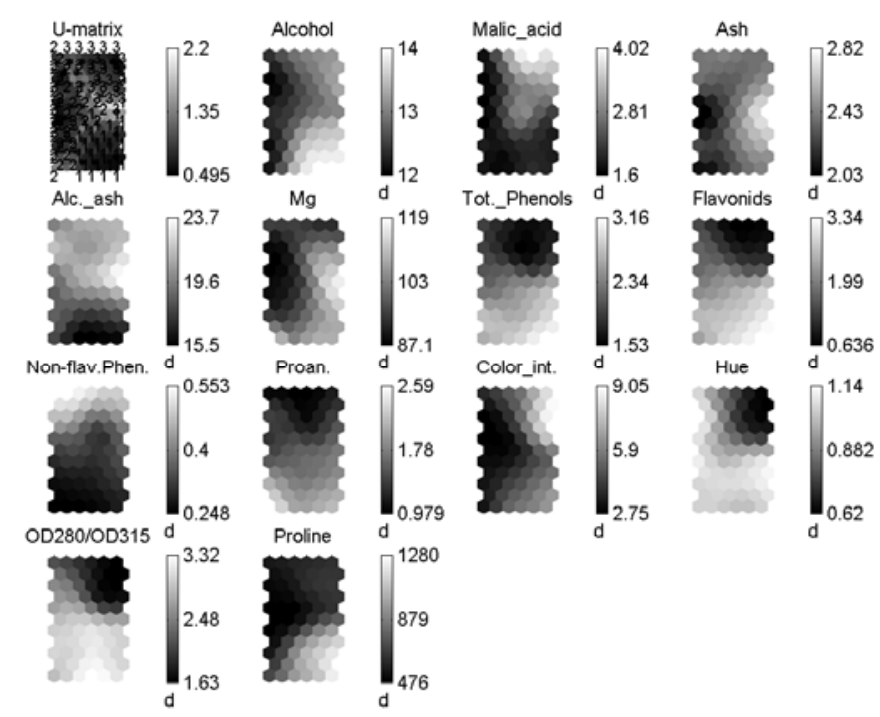

Fig. 6. Self-Organizing Map of the Wine data

As can be seen from Table 2, because of the simplicity of the proposed clustering algorithm, the proposed approach is attractive in comparison with other iterative and optimization schemes that involves extensive intermediate optimization to generate fuzzy classifiers.

The results are summarized in Table 2.As it is shown, the performance of the obtained classifiers are comparable to those in [37] and [13], but use far less rules (3-5 compared to 60) and less features.

Table 2. Classification rates on the Wine data for ten independent runs.

\begin{tabular}{lccccc}
\hline Method & Best result & Aver result & Worst result & Rules & Model eval \\
\hline Corcoran and Sen [37] & $100 \%$ & $99.5 \%$ & $98.3 \%$ & 60 & 150000 \\
Ishibuchi et al. [13] & $99.4 \%$ & $98.5 \%$ & $97.8 \%$ & 60 & 6000 \\
\hline Cluster + GA & $99.4 \%$ & varying schemes & $98.3 \%$ & 3 & $4000-8000$ \\
\hline Gath-Geva clustering & $95.5 \%$ & $95.5 \%$ & $95.5 \%$ & 3 & 1 \\
\hline Sup. cluster (13 features) & $98.9 \%$ & $98.9 \%$ & $98.9 \%$ & 3 & 1 \\
Sup. cluster (5 features) & $100 \%$ & $100 \%$ & $100 \%$ & 5 & 2 \\
\hline
\end{tabular}

Comparing the fuzzy sets in Figure 5 with the data in Figure 3 shows that the obtained rules are highly interpretable. For example, the Flavonoids are divided in Low, Medium and High, which is clearly visible in the data. This knowledge can be easily validated by analyzing the SOM of the data given in Figure 6 


\section{Conclusion}

The design of rule base classifiers is approached by combining a wide range of CI tools developed for knowledge representation (fuzzy rules), feature selection (class separability criterion, model initialization (clustering and decision tree), model reduction (orthogonal methods) and tuning (genetic algorithm). It has been shown that these tools can be applied in a synergistic manner though the nine steps of knowledge discovery.

\section{References}

1. U. Fayyad, G. Piatestku-Shapio, P. Smyth, R. Uthurusamy, Advances in Knowledge Discovery and Data Mining, AAAI/MIT Press, 1996.

2. R. Brachman, T. Anand, The process of knowledge discovery in databases, in: Advances in Knowledge Discovery and Data Mining, AAAI/MIT Press, 1994, pp. 37-58.

3. U. Fayyad, G. Piatestku-Shapio, P. Smyth, Knowledge discovery and data mining: Towards a unifying framework, in: Advances in Knowledge Discovery and Data Mining, AAAI/MIT Press, 1994.

4. J. Abonyi, R. Babuska, H. Verbruggen, F. Szeifert, Using a priori knowledge in fuzzy model identification, International Journal of Systems Science 31 (2000) 657-667.

5. J. Abonyi, L. Nagy, F. Szeifert, Hybrid fuzzy convolution modelling and identification of chemical process systems, International Journal of Systems Science 31 (2000) 457-466.

6. J. Abonyi, A. Bodizs, L. Nagy, F. Szeifert, Hybrid fuzzy convolution model and its application in predictive control, Chemical Engineering Research and Design 78 (2000) 597-604.

7. J. Mao, K. Jain, Artificial neural networks for feature extraction and multivariate data projection, IEEE Trans. on Neural Networks 6(2) (1995) 296-317.

8. S. Abe, R. Thawonmas, Y. Kobayashi, Feature selection by analyzing regions approximated by ellipsoids, IEEE Trans. on Systems, Man, and Cybernetics, Part. C 28(2) (1998) 282-287.

9. J. V. de Oliveira, Semantic constraints for membership function optimization, IEEE Trans. FS 19 (1999) 128-138.

10. M. Setnes, R. Babuška, Fuzzy relational classifier trained by fuzzy clustering, IEEE Trans. on Systems, Man, and Cybernetics, Part. B 29 (1999) 619-625.

11. R. Setiono, Generating concise and accurate classification rules for breast cancer diagnosis, Artificial Intelligence in Medicine 18 (2000) 205-219.

12. D. Nauck, R. Kruse, Obtaining interpretable fuzzy classification rules from medical data, Artificial Intelligence in Medicine 16 (1999) 149-169.

13. H. Ishibuchi, T. Nakashima, T. Murata, Performance evaluation of fuzzy classifier systems for multidimensional pattern classification problems, IEEE Trans. SMC-B 29 (1999) 601-618.

14. M. Setnes, J. Roubos, Rule-based modeling: Precision and transparency, IEEE Trans. FS. in press.

15. L. Sethi., Entropy nets: From decision trees to neural networks, Proc. IEEE 78 (1990) 1605-1613.

16. I. Ivanova, M. Kubat, Initialization of neural networks by means of decision trees, Knowledge-Based Systems 8 (1995) 333-344. 
17. R. Setiono, W. Leow, On mapping decision trees and neural networks, Knowledge Based Systems 13 (1999) 95-99.

18. J.-S. Jang, C.-T. Sun, Functional equivalence between radial basis function networks and fuzzy inference systems, IEEE Trans. NN 4 (1993) 156-159.

19. L. T. Kóczy, D. Tikk, T. D. Gedeon, On functional equivalence of certain fuzzy controllers and rbf type approximation schemes, International Journal of Fuzzy Systems .

20. T. Kohonen, The self-organizing map, Proceedings of the IEEE 78(9) (1990) 1464-1480.

21. J. Abonyi, F. Szeifert, Supervised fuzzy clustering for the identification of fuzzy classifiers, in: 6th Online World Conference on Soft Computing in Industrial Applications, 2001.

22. M. Setnes, R. Babuška, U. Kaymak, H. van Nauta Lemke, Similarity measures in fuzzy rule base simplification, IEEE Trans. SMC-B 28 (1998) 376-386.

23. M. Setnes, J. Roubos, Transparent fuzzy modeling using fuzzy clustering and GA's, in: In NAFIPS, New York, USA, 1999, pp. 198-202.

24. J. Roubos, M. Setnes, Compact fuzzy models through complexity reduction and evolutionary optimization, in: Proc. of IEEE international conference on fuzzy systems, San Antonio, USA, 2000, pp. 762-767.

25. Y. Yam, P. Baranyi, C. Yang, Reduction of fuzzy rule base via singular value decomposition, IEEE Trans. Fuzzy Systems 7(2) (1999) 120-132.

26. J. Yen, L. Wang., Simplifying fuzzy rule-based models using orthogonal transformation methods, IEEE Trans. SMC-B 29 (1999) 13-24.

27. M. Setnes, R. Babuška, Rule base reduction: Some comments on the use of orthogonal transforms, IEEE Trans. on Systems, Man, and Cybernetics, Part. B 31 (2001) 199-206.

28. K. Cios, W. Pedrycz, R. Swiniarski, Data Mining Methods for Knowledge Discovery, Kluwer Academic Press, Boston, 1998.

29. J.A.Roubos, M. Setnes, J. Abonyi, Learning fuzzy classification rules from data, in: Developments in Soft Computing, 2000, pp. 108-115.

30. Y. Jin, Fuzzy modeling of high-dimensional systems, IEEE Trans. FS 8 (2000) 212-221.

31. J. Roubos, M. Setnes, J. Abonyi, Learning fuzzy classification rules from data, in: RASC conference, Leichester, UK, 2000.

32. J. Abonyi, R. Babuška, F. Szeifert, Fuzzy modeling with multidimensional membership functions: Grey-box identification and control design, IEEE Trans. on Systems, Man, and Cybernetics, Part. C (2001) In Print.

33. J. Abonyi, J. Roubos, Structure identification of fuzzy classification rules, in: 5th Online World Conference on Soft Computing in Industrial Applications (WSC5), 2000.

34. F. Hoppner, F. Klawonn, R. Kruse, T. Runkler, Fuzzy Cluster Analysis - Methods for Classification, Data Analysis and Image Recognition, John Wiley and Sons, 1999.

35. O. Nelles, M. Fischer, Local linear model trees (LOLIMOT)for nonlinear system identification of a cooling blast, in: European Congress on Intelligent Techniques and Soft Computing (EUFIT), Aachen, Germany, 1996.

36. J.-S. Jang, Structure determination in fuzzy modeling: A fuzzy cart approach, in: Proc. of IEEE international conference on fuzzy systems, Orlando, USA, 1994.

37. A. Corcoran, S. Sen, Using real-valued genetic algorithms to evolve rule sets for classification, in: IEEE-CEC, Orlando, USA, 1994, pp. 120-124. 